\title{
Measurement of Beam Lifetime and Applications for SPEAR3
}

\author{
Xiaobiao Huang, Jeff Corbett \\ SLAC National Accelerator Laboratory, 2575 Sand Hill Road, Menlo Park, CA 94025
}

\begin{abstract}
Beam lifetime studies for the SPEAR3 storage ring are presented. The three lifetime components are separated with lifetime measurements under various combinations of beam currents and fill patterns and vertical scraper scans. Touschek lifetime is studied with rf voltage scans and with the horizontal or vertical scrapers inserted. The measurements are explained with calculations based on the calibrated lattice model. Quantum lifetime measurements are performed with reduced longitudinal and horizontal apertures, respectively, from which we deduce the radiation energy loss down to a few keV per revolution and the horizontal beam size.
\end{abstract}

Keywords: beam, lifetime, SPEAR3

\section{Introduction}

In an electron storage ring, the stored beam current slowly diminishes because electrons are constantly lost to the finite aperture. There are four major loss mechanisms: elastic collisions between electrons and residual gas atoms (Coulomb scattering), inelastic collisions between electrons and residual gas atoms (bremsstrahlung), collisions between electrons (Touschek scattering) and photon emission (quantum lifetime) [1]. Since the four mechanisms have distinct signatures based on physical machine and dynamical beam parameters, such as rf gap voltage, residual gas pressure, momentum compaction factor, limiting apertures and beam size, measurement of beam lifetime under various conditions is an important tool to characterize key storage ring and machine performance parameters.

Email address: xiahuang@slac.stanford.edu (Xiaobiao Huang)

Preprint submitted to Nuclear Inst. and Methods in Physics Research, AOctober 20, 2010 
In this paper we report on studies at SPEAR3 designed to characterize the beam lifetime components and to confirm machine parameters calculated with a particle tracking model. SPEAR3 is a 3rd-generation, double-bend achromat electron storage ring with a circumference of $234.1 \mathrm{~m}$. The rf harmonic number is 372 . At a beam energy of $3 \mathrm{GeV}$ the horizontal emittance is $10 \mathrm{~nm}$-rad in the low emittance optics when insertion device radiation effects are taken into account. For a $100 \mathrm{~mA}$ stored beam, the lifetime is typically $40 \sim 50$ hours at $\sim 0.2 \%$ coupling.

Measurement of beam lifetime for SPEAR3 is briefly described in section 2. In section 3 we discuss the method used to separate the three lifetime components for SPEAR3. In section 4 we present Touschek lifetime measurements and associated calculations based on different beam scraper conditions. Quantum lifetime measurements and their applications are described in section 5. Section 6 provides a short summary of the results.

\section{Beam Lifetime Measurements}

Beam lifetime measurements in SPEAR3 are typically achieved by fitting the dc current transformer (DCCT) data as a linear function of time over short time intervals. The accuracy of lifetime measurement depends on the accuracy of the DCCT and the data duration period. It can be shown that

$$
\frac{\sigma_{\tau}}{\tau}=\frac{2 \sqrt{3}}{\sqrt{N}} \frac{\sigma_{I}}{\Delta I},
$$

where $\tau$ and $\sigma_{\tau}$ are the beam lifetime and its uncertainty, respectively, $N$ is the number of acquired data points, $\Delta I$ the current loss in the data acquisition period and $\sigma_{I}$ the uncertainty or noise on the DCCT data. For SPEAR3, the uncertainty of a one-second average is about $1 \mu \mathrm{A}$ so that for a beam with $100 \mathrm{~mA}$ total current and 50 hrs lifetime, it takes 75 seconds of data acquisition to achieve a $1 \%$ error bar on the lifetime measurement.

In an given experimental arrangement the beam lifetime may decrease significantly as the beam conditions change. In such cases, the DCCT data duration is reduced (but no less than 20 seconds) to maintain roughly the same accuracy. When the beam lifetime is very short, the DCCT data are fit with exponential curves as the linear model is no longer a valid approximation. 


\section{Gas Scattering}

The three main beam loss processes can be studied according to their characteristic dependence on the physical machine and dynamical beam parameters. For example, the Coulomb and bremsstrahlung loss rates are proportional to the residual gas pressure, but are independent of beam size. The Coulomb loss rate is inversely proportional to the transverse beam acceptance, but is independent of the momentum acceptance. The Touschek loss rate on the other hand is independent of residual gas pressure, but is inversely proportional to beam size. The Touschek loss rate depends strongly on the momentum acceptance, but the bremsstrahlung loss rate is only weakly dependent on the momentum acceptance.

Quantum lifetime contribution becomes visible only when the acceptance is smaller than about seven times of the beam dimension [1]. Since for most machines the transverse and longitudinal acceptances are larger than ten times the beam size, quantum lifetime is negligible for normal operation conditions.

To determine the individual contributions of the three main loss mechanisms under nominal conditions, we conducted two types of experiments. In the first type, beam lifetime was measured under various combinations of the total beam current and the total number of bunches in the ring. For each of five fill patterns with 20, 40, 80, 140, 280 bunches, respectively, the ring was filled with 25, 50, 75, $100 \mathrm{~mA}$, resulting in 20 different conditions for which the lifetime was measured to an accuracy of $2 \%$ or better.

The vacuum pressure in the ring has a base value of $\sim 1 \mathrm{nT}$ and varies in proportion to the total beam current due to neutral gas desorption from the vacuum chamber walls. Figure 1 shows the recorded average vacuum pressure vs. total current. A linear model of the pressure as a function of total current was used to fit the data, including finite base pressure.

The total electron beam loss rate can be split into two parts including gas scattering losses (Coulomb and bremsstrahlung) proportional to vacuum pressure, and intrabeam or Touschek scattering proportional to single bunch current, i.e.,

$$
\frac{1}{\tau}=a P(I)+b I_{b} / r_{z}\left(I_{b}\right)
$$

where $a$ and $b$ are fitting coefficients, vacuum pressure $P(I)$ is given by the fitted linear model and $I_{b}$ is the single bunch current. An explicit bunch lengthening factor $r_{z}=\frac{\sigma_{z}}{\sigma_{z 0}}$ is also included to account for inductive impedance and 
$\sigma_{z 0}$ is the zero-current bunch length. The empirical bunch lengthening model in Ref. [2] is used to calculate the bunch length. Eq. (2) fits the data very well, with differences between the model and measurements less than $5 \%$ for most data points excluding two outliers. According to the fitting result, the gas scattering lifetime is $145 \pm 3 \mathrm{hrs}$ and the Touschek lifetime is $60.1 \pm 0.3 \mathrm{hrs}$ for a nominal operation condition of $100 \mathrm{~mA}$ stored current in 280 bunches.

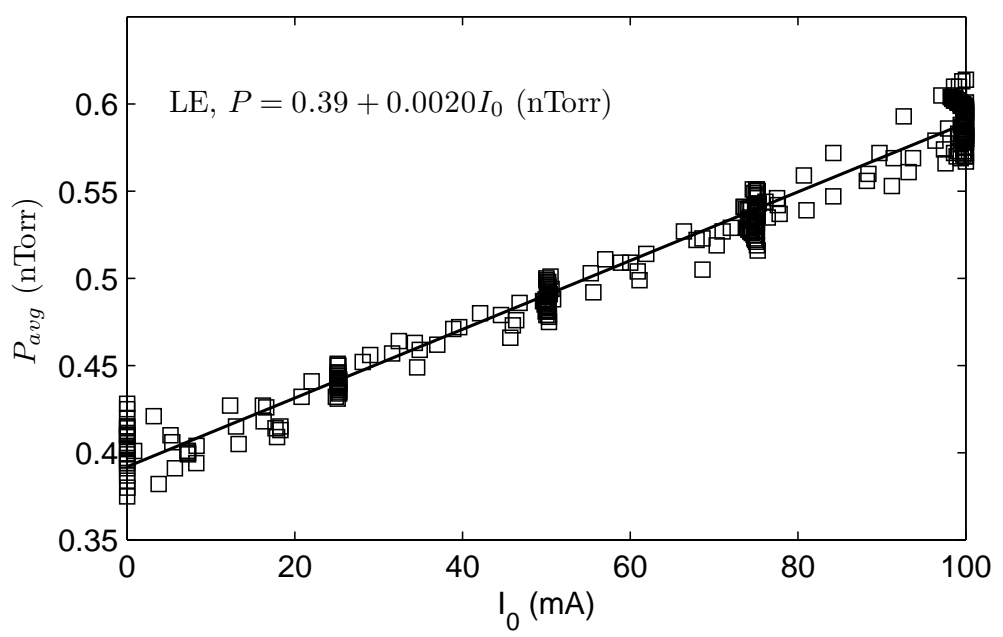

Figure 1: Dynamic neutral gas pressure increase with beam current.

The Coulomb lifetime can be separated from the combined gas scattering loss component by measuring beam lifetime with a vertical scraper inserted to a series of different positions. Figure 2 shows the raw scan data taken when the ring was filled to $100 \mathrm{~mA}$ in 280 bunches before the scans started. As the vertical scraper moves in, initially the lifetime does not change because the scraper is shielded by other vertical limiting apertures in the ring. As the scraper becomes the limiting aperture, the Coulomb lifetime component changes quadratically with respect to the vertical scraper aperture. The Touschek lifetime and bremsstrahlung lifetime may also change if the reduced vertical aperture causes the momentum aperture to decrease.

The total beam current in these scraper scans decreased less than $2 \%$ from the start to the point when the scraper is $1.1 \mathrm{~mm}$ away from the beam. However, lifetime vs. rf gap voltage measurements, with the vertical scraper inserted show no reduction of momentum aperture until the scraper-to-beam distance is $0.7 \mathrm{~mm}$ or less (see section 4). The Touschek 
lifetime and bremsstrahlung lifetime contributions are therefore constant for this period and the data may be described by the model

$$
\frac{1}{\tau}=\frac{1}{\tau_{T B}}+\frac{1}{\tau_{C}} \frac{a_{0}^{2}}{\min \left(a_{0}^{2}, y^{2}\right)},
$$

where $\tau_{T B}$ is the combined Touschek and bremsstrahlung lifetime, $\tau_{C}$ the Coulomb lifetime at the start of the scan, $a_{0}$ the vertical ring aperture at the scraper location and $y$ the scraper distance from the beam center. Coulomb loss on the horizontal aperture is neglected since the horizontal acceptance is much larger than the vertical acceptance [1]. The average ratio of the maximum allowed vertical scattering angle to the maximum horizontal angle is estimated to be 0.28 for SPEAR3.

Fitted curves are also shown in Figure 2. The fitted parameters result in $\tau_{T B}=49.2 \pm 0.3 \mathrm{hrs}, \tau_{C}=339 \pm 16 \mathrm{hrs}, a_{0}=4.11 \pm 0.09 \mathrm{~mm}$. Combining this result with the fitted results from Eq. (2), we obtain the separate contributions for the three lifetime components as shown in Table 1. The linear optics for the lattice during the experiments was measured with the orbit response matrix method (LOCO) [3]. The resulting linear coupling ratio $\left(\epsilon_{y} / \epsilon_{x}\right)$ estimated from the LOCO calculation is also listed in the table.

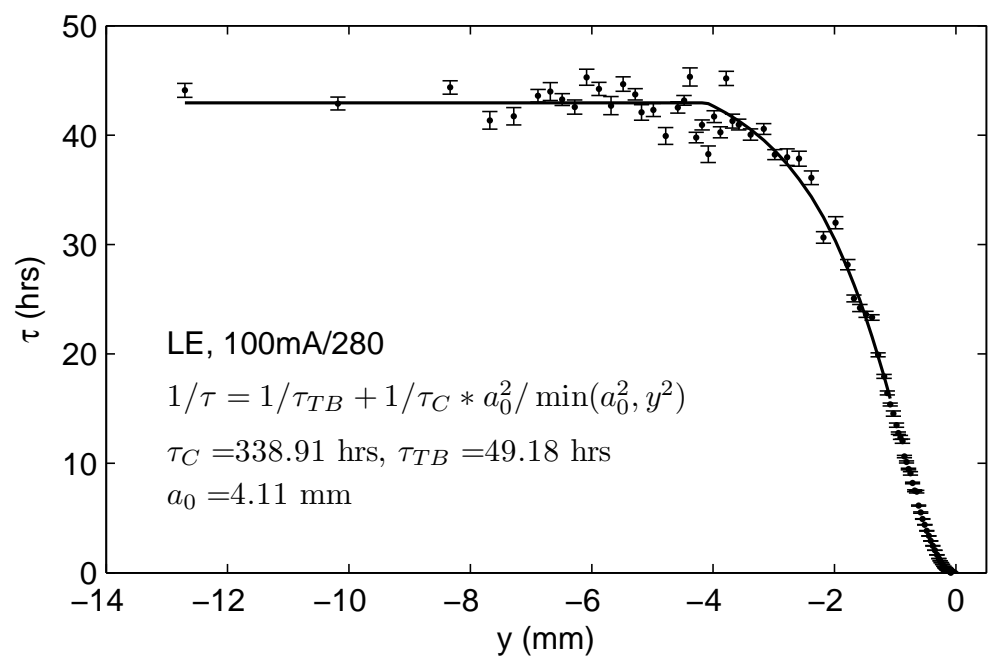

Figure 2: Beam lifetime vs. vertical scraper position. The ring was filled $100 \mathrm{~mA}$ in 280 bunches. 
Table 1: Lifetime components for SPEAR3 at $100 \mathrm{~mA}, 280$ bunches.

\begin{tabular}{|l|c|c|c|c|c|}
\hline Lattice & Touschek & Coulomb & bremstr. & Total & $\epsilon_{y} / \epsilon_{x}$ \\
\hline Low emit. & 60.3 & 332 & 262 & 42.7 & $0.112 \%$ \\
\hline
\end{tabular}

\section{Touschek Lifetime}

\subsection{Calculation of Touschek Lifetime}

Touschek lifetime is usually the dominate beam loss mechanism for modern 3rd-generation light sources because of the small emittance and high single bunch current. The loss rate for Touschek scattering is given by [1]

$$
\frac{1}{\tau_{\mathrm{T}}}=\frac{N r_{0}^{2} c}{16 \gamma^{2} \pi \sigma_{z}}\left\langle\frac{1}{\sigma_{x} \sigma_{y}}\left(\frac{D\left(\xi_{+}\right)}{\delta_{\mathrm{m}+}^{3}}+\frac{D\left(\xi_{-}\right)}{\delta_{\mathrm{m}-}^{3}}\right)\right\rangle,
$$

where $\langle\cdot\rangle$ represents an average over the ring circumference, $N$ is the number of electrons in the bunch, $r_{0}=2.82 \times 10^{-15} \mathrm{~m}$ the classical electron radius, $\gamma$ the Lorentz factor, $\sigma_{x, y, z} \mathrm{rms}$ sizes for the three directions, $\xi_{ \pm}=\left(\delta_{\mathrm{m} \pm} / \gamma \sigma_{x^{\prime}}\right)^{2}$, $\sigma_{x^{\prime}}$ the rms horizontal divergence, $\delta_{\mathrm{m} \pm}$ the high and low limits of the momentum aperture, respectively. $D(\xi)$ is a slowly varying function of $\xi$. Eq. (4) shows that Touschek loss rate is directly proportional to the number density of electrons of the bunch in physical space. While the longitudinal beam size $\sigma_{z}$ is considered invariant around the ring, the transverse sizes change substantially from one location to another according to their corresponding beta functions. The vertical beam size depends strongly on the linear coupling between the $x-y$ planes.

The momentum aperture is defined as the maximum energy error an inelastically scattered electron can have without being lost. The electron will be lost if its energy error is outside of the rf bucket or if it acquires large transverse offset due to off-momentum betatron motion [4]. The momentum aperture as determined by transverse motion is dependent on the local horizontal beta function and dispersion. Theoretical calculations of the local momentum aperture are difficult since at large amplitude the beam motion is usually dominated by nonlinearities of the dynamic system. Tracking particles in 6D phase space for many turns is therefore the usual method for accurate determination of the momentum aperture $[5,6]$. Figure 3 shows for example the change in momentum aperture as tracked throughout the 
SPEAR3 magnet lattice with the horizontal scraper inserted to a series of different positions, from $-25 \mathrm{~mm}$ to $-3 \mathrm{~mm}$.

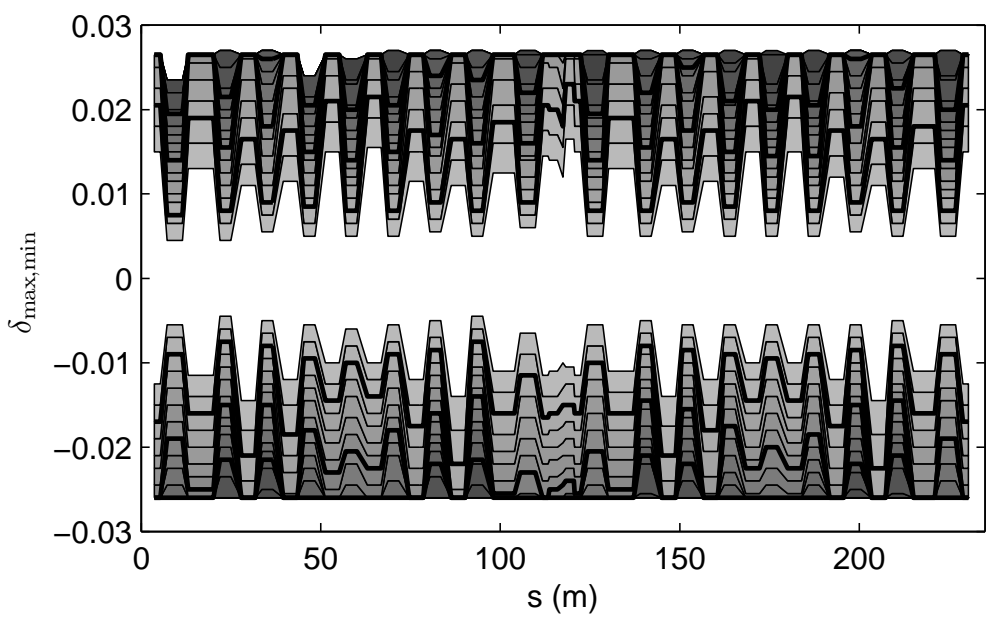

Figure 3: Momentum aperture with the horizontal scraper inserted to various positions. The three thick lines are for $x=-15,-10$ and $-5 \mathrm{~mm}$, respectively.

Knowing the momentum aperture and the linear optics of a storage ring, the Touschek lifetime can be estimated from Eq. (4). The calculated Touschek lifetime corresponding to experimental conditions in Table 1 is 53.0 hrs.

\subsection{Measurement of Touschek Lifetime}

A commonly used technique to characterize Touschek lifetime in a storage ring is to measure the rate of beam decay under various rf gap voltage levels [4]. As the rf gap voltage decreases, the rf bucket height also decreases. When the bucket height defines the momentum aperture for the entire ring, the Touschek lifetime will decrease with rf gap voltage. Conversely, if the local momentum aperture as determined by transverse betatron motion is smaller than the bucket height (such as when the horizontal scraper inserted), decreasing the rf gap voltage will cause the Touschek lifetime to increase as the bunches become longer reducing charge density. In the more complicated case when the local momentum aperture is given by the bucket height for a portion of the ring and by the transverse aperture for the rest of the ring, the Touschek lifetime dependence on the rf gap voltage will be a mixture of the above two cases. 
Figure 4 shows the electron beam lifetime as a function of $\mathrm{rf}$ gap voltage in SPEAR3 with the horizontal scraper at different positions. For these measurements $100 \mathrm{~mA}$ beam current was stored in 20 bunches to enhance the Touschek scattering rate. In the normal case when the scraper is pulled out, the rf bucket height defines the momentum aperture for the entire ring. This indicates that the dynamic aperture of the ring as determined by the nonlinear dynamics is sufficiently large to contain Touschek particles. As the scraper is moved in, the local momentum aperture shrinks and starts to affect the Touschek lifetime. Figure 4 also shows the calculated Touschek lifetime under the experimental condition with the tracked momentum aperture. There is good agreement between the measurement and modeling.

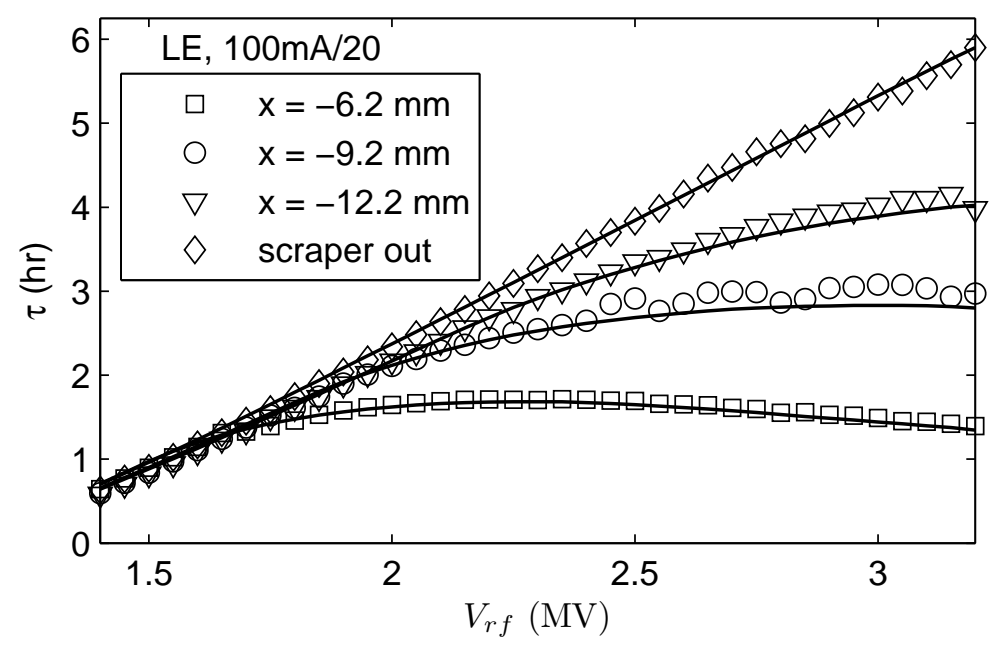

Figure 4: Touschek lifetime vs. rf gap voltage with the horizontal scraper out or inserted at various positions for $100 \mathrm{~mA}$ current in 20 bunches. Error bars are below 1\%. The coupling coefficient was $0.19 \%$. Dark lines show calculations for the corresponding cases.

The rf voltage scan measurements were also made with the vertical scraper inserted (Figure 5). For this case the measured Touschek lifetime was obtained by subtracting the Coulomb contribution from the total lifetime according to the vertical scraper scan measurement in the previous section. It is observed that before the scraper tip gets very close to the beam $(<1 \mathrm{~mm})$, it has little effect on the Touschek lifetime. When the scraper is $0.7 \mathrm{~mm}$ away from the beam, the measured Touschek lifetime vs. rf gap voltage curve is approximated very well by the calculated curve assuming the horizontal scraper 
is at $-15.5 \mathrm{~mm}$ away from the beam. Touschek particles initially only have large momentum error and therefore large horizontal oscillation amplitudes. But with large oscillation amplitude, the horizontal motion can couple to the vertical plane and be lost at vertical apertures [4]. From Figure 5 it is seen that Touschek particles with horizontal amplitude larger than $15.5 \mathrm{~mm}$ at the scraper location do couple to vertical motion with oscillation amplitude above $0.7 \mathrm{~mm}$.

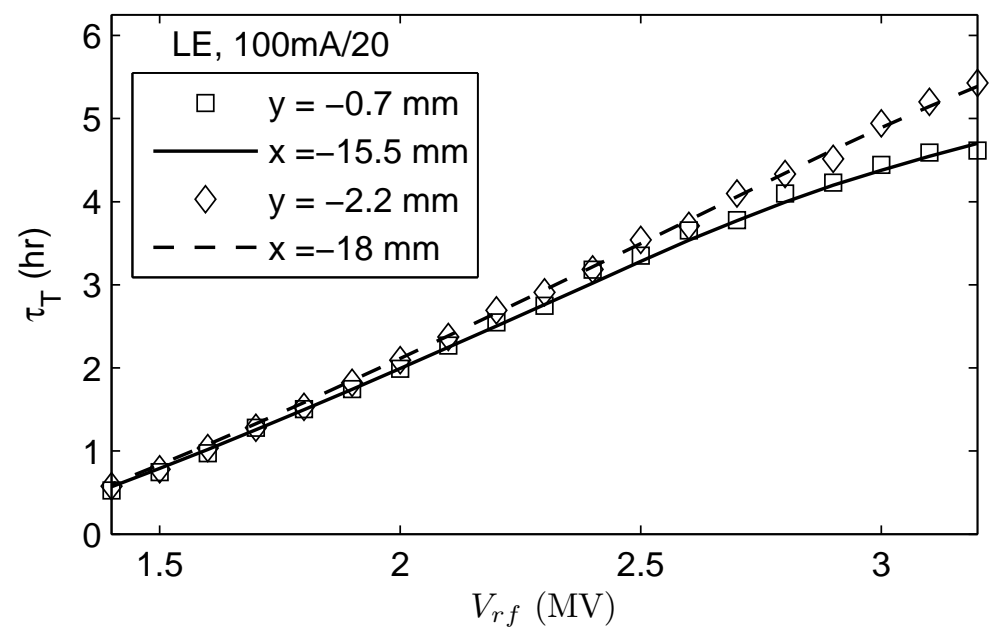

Figure 5: Touschek lifetime vs. rf gap voltage with the vertical scraper out or inserted and $100 \mathrm{~mA}$ current in 20 bunches. Error bars are below 1\%. The solid line shows calculated result with the horizontal scraper at $-15.5 \mathrm{~mm}$.

We further tested the horizontal/vertical coupling hypothesis by a tracking simulation which included correct coupling and insertion device nonlinearities. In the simulation Touschek particles are launched from an arc location and observed at the scraper. The maximum vertical amplitude is plotted against the maximum horizontal offset toward the negative side in Figure 6. The two branches of the simulated data represent negative and positive energy errors, respectively. Again, the simulation agrees with the experimental observation very well.

By measuring Touschek lifetime vs. rf voltage curves with the vertical scraper at various positions and approximating the data with curves that correspond to a series of horizontal scraper positions, we may establish a mapping relationship between the maximum horizontal oscillation amplitude 


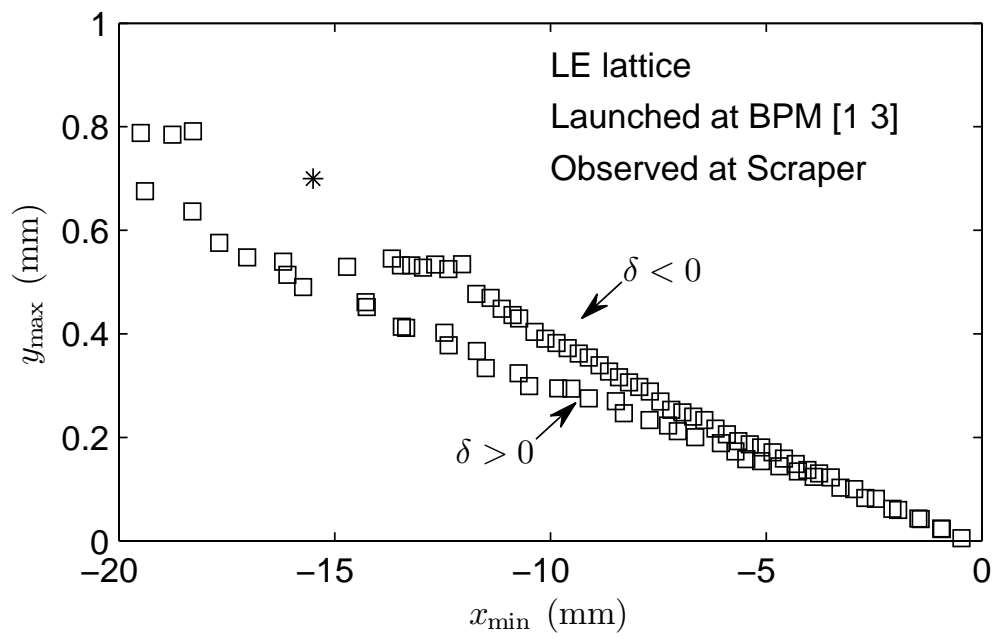

Figure 6: Maximum vertical amplitude vs. horizontal offset at the scraper position for Touschek particles launched from an arc location. The experimental data is also shown by the ' $*$ '.

and the maximum vertical amplitude for Touschek scattered particles. The mapping serves as a global characterization of the nonlinear dynamics in the storage ring and is useful for determining both radii needed for beam collimation and vacuum chamber size for future insertion devices.

\section{Quantum Lifetime Measurement}

Normally the electron bunches in a storage ring have a Gaussian distribution in all three dimensions. When the aperture of the ring is substantially reduced in any dimension, the tails of the Gaussian distribution can be scraped, leading to beam loss. Quantum beam loss occurs when stochastic photon emission drives individual electrons into the tails or 'halo' of the distribution. The quantum contribution to beam lifetime can be calculated by [1]

$$
\tau_{q}=\tau_{w} \frac{e^{u}}{2 u}, \text { with } u=\frac{A^{2}}{2 \sigma^{2}},
$$

where $\tau_{w}, A$ and $\sigma$ are the damping time, aperture and rms beam size, respectively for any of the horizontal, vertical and longitudinal directions. 
Direct measurements of quantum beam lifetime may serve as a probe into some of the vital beam parameters. At SPEAR3, for instance, quantum lifetime measurements were used to measure the radiative beam energy loss from insertion devices and the horizontal beam size.

\subsection{Measurement of Insertion Device Energy Loss}

The rf bucket height becomes the momentum aperture when the rf acceleration voltage is sufficiently reduced. Measurement of quantum lifetime with reduced rf voltage can be used to determine the actual bucket height, which in turn can be used to calculate the total beam energy loss per revolution. The rf bucket height is given by [7]

$$
\delta_{\max }=\left(\frac{2}{\pi h \alpha} \frac{U_{0}}{E}\right)^{1 / 2}\left(\sqrt{q^{2}-1}-\arccos \frac{1}{q}\right)^{1 / 2},
$$

where $h$ is the harmonic number, $\alpha$ the momentum compaction factor, $E$ the beam energy, $U_{0}$ the total energy loss per revolution, $q=e V_{\mathrm{rf}} / U_{0}$ and $V_{\mathrm{rf}}$ the peak rf voltage. The longitudinal damping time depends on the total rate of beam energy loss. Since the bend magnet field in a storage ring is usually well calibrated, we can use the calculated energy loss in the bending magnets as a starting point. Defining the ratio of insertion device energy loss (or any losses other than bend magnet radiation) over the calculated bending magnet energy loss as $\Delta=U_{\mathrm{ID}} / U_{\mathrm{B}}$, the longitudinal damping rate is given by

$$
\frac{1}{\tau_{z}(\Delta)}=\frac{U_{\mathrm{B}}}{T_{0} E}\left(1+\Delta+\frac{\mathcal{D}_{\mathrm{B}}}{2}\right),
$$

where $T_{0}$ is the revolution period, $\mathcal{D}_{\mathrm{B}}=I_{4} / I_{2}$ with radiation integrals $I_{2}$ and $I_{4}$ calculated with bending magnets only [7]. Theoretically the momentum spread is also altered by insertion device radiative loss. However, such changes are usually small, for example, only $0.5 \%$ of the nominal value of $\sigma_{E}$ when all insertion devices are included for SPEAR3. In this study we use the calculated momentum spread assuming bend magnet radiation only, $\sigma_{E}=0.97 \times 10^{-3}$. The calculated bend magnet radiation loss is $U_{\mathrm{B}}=0.881 \mathrm{MeV}$.

The quantum lifetime was then measured with the insertion devices in four states: all IDs closed, all IDs closed but BL6 ID fully open, all IDs closed but BL7 ID fully open and all IDs fully open (Figure 7). The measurements 
were repeated for each case 2 to 3 times. Touschek lifetime was first calculated and deducted from the total lifetime to obtain the quantum lifetime. To avoid errors in the Touschek lifetime calculation, we kept only the data points for which the Touschek contribution was less than $15 \%$ of the total loss rate. A characteristic curve showing $u=A^{2} / 2 \sigma^{2}$ as a function of $\Delta=U_{\mathrm{ID}} / U_{\mathrm{B}}$ was then constructed by solving the first equation in Eq. (5) with $\Delta$ covering the candidate range. The second relation in Eq. (5) gives another $u \sim \Delta$ curve with $A$ replaced by $\delta_{\max }$, and $\sigma$ by $\sigma_{E}$. The energy loss difference from the bare lattice bend magnet radiation $U_{\mathrm{B}}$ (or the $\Delta$ parameter) is determined by the intersection of the two curves. This is illustrated by Figure 8, where data for the case with BL6 open are used. The lower 'flat' curves are derived from the quantum lifetime data and the upper 'tilted' curves are from the bucket height calculations. For the four data points shown, the resulting values of $\Delta \approx 0.25$ are remarkably close despite the large differences in measured lifetime. Hence the radiated ID power is about $220 \mathrm{keV} /$ turn.

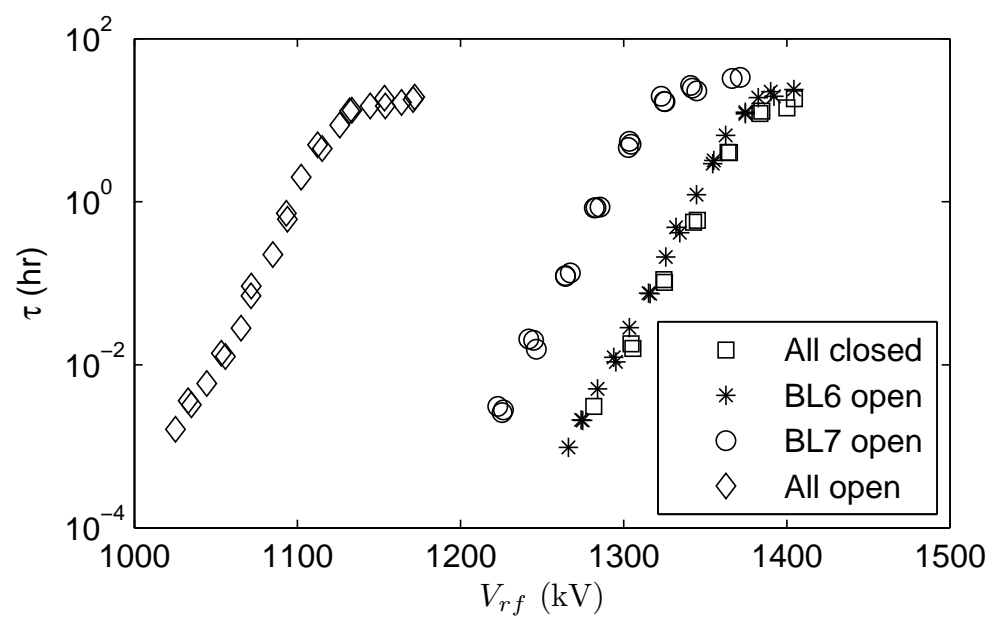

Figure 7: Measured lifetime vs. rf gap voltage in quantum lifetime regime with four ID states. The ring was filled $17 \mathrm{~mA}$ in 280 bunches for all cases.

The measured energy loss for all four cases was compared with the calculated values in Table 2 . The measured values and the error bars are based on the averages and standard deviations of around 10 data points under the same experimental conditions. Notice that the small calculated $\Delta$ for the all IDs open case in the table is not zero. This is because the nominal bend 


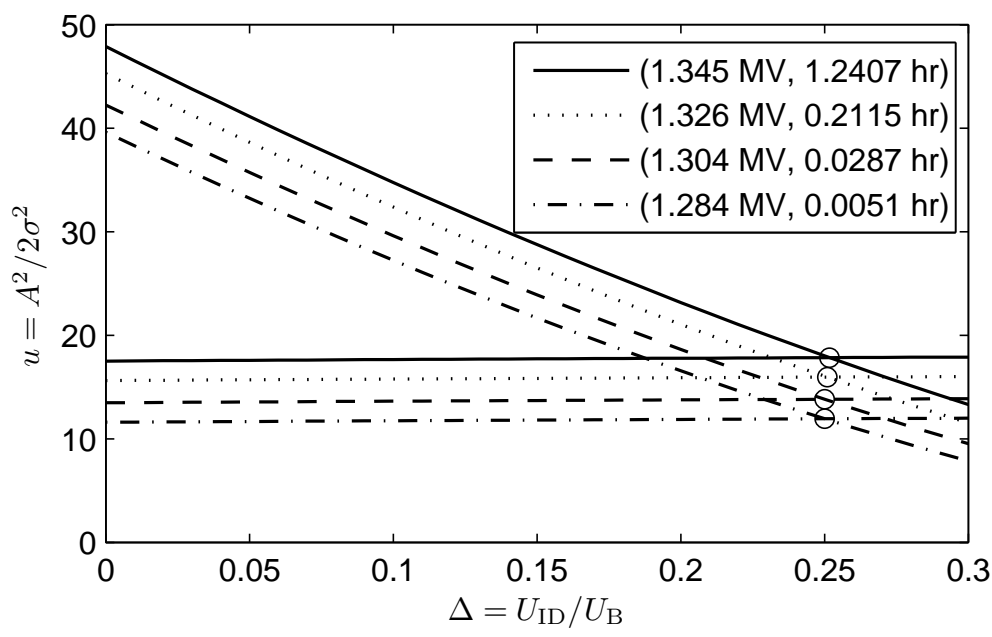

Figure 8: Solution for $\Delta$ by finding the intersection of two $u \sim \Delta$ curves obtained from Eq. (5). Data are for the case with all but BL6 ID closed. The legend shows the corresponding rf gap voltage and quantum lifetime data for the four curves.

magnet parameters are based on hard-edge sector dipole models. In reality SPEAR3 dipole are gradient dipoles with straight geometry where the bending radius varies along the reference orbit. This effect and the fringe field effect are both included in a magnet model discussed in [8]. The corrections to the radiation integral $I_{2}$ are included in all calculated values in Table 2 .

The insertion device radiation calculations are all based on sinusoidal field models and are in good agreement with the measured values. Comparing measurement and calculation results for the case with all IDs open and the measured results for the cases with the BL6 ID open or closed while all other IDs were closed, it is clear that this technique can resolve small changes in energy loss, down to the $4 \mathrm{keV}$ level.

Table 2: Comparison of measured radiative energy loss to model calculations. Numbers in parentheses indicate the uncertainty of the last two digits.

\begin{tabular}{|l|c|c|c|c|}
\hline ID status & all closed & BL6 open & BL7 open & all open \\
\hline meas $\Delta$ & $0.2583(12)$ & $0.2511(14)$ & $0.1919(25)$ & $-0.0052(23)$ \\
calc $\Delta$ & 0.2603 & 0.2515 & 0.2048 & -0.0039 \\
\hline
\end{tabular}




\subsection{Measurement of the Horizontal Beam Size}

When a horizontal scraper is inserted near the beam core, quantum losses will dominate the electron beam lifetime. Experiments were carried out for SPEAR3 under two lattices: the nominal operation lattice (low emittance) and the achromatic lattice with zero dispersion in straight sections and a horizontal emittance of $15 \mathrm{~nm}$-rad with all insertion devices closed. The ring was filled with one 'giant' bunch containing $28 \mathrm{~mA}$ circulating current for the low emittance lattice and $23 \mathrm{~mA}$ in the achromatic lattice for the horizontal scraper scans. As the horizontal aperture was reduced, both the gas scattering lifetime and Touschek lifetime decreased as expected with Touschek lifetime dominating before quantum lifetime set in. In the region where quantum lifetime is significant, the total beam lifetime can be fitted with the combination of Touschek and quantum lifetime.

When the physical aperture is small, the local momentum aperture may be approximated by $\delta_{\max }(s)=A_{x 0} /\left(\sqrt{\beta_{0} \mathcal{H}(s)}+D_{0}\right)$, where $A_{x 0}, \beta_{0}$ and $D_{0}$ are the horizontal aperture, beta function and dispersion at the scraper location and $\mathcal{H}=\gamma D^{2}+2 \alpha D D^{\prime}+\beta D^{\prime 2}$. The $\mathcal{H}$ function for SPEAR3 is nearly a binary function with different values in the arc and straight sections. The Touschek loss rate may therefore be considered as the sum of a constant term and a term proportional to $1 / A_{x 0}^{3}$. The total loss rate may be fitted with a model

$$
\frac{1}{\tau}=\left(\frac{1}{\tau_{1}}+\frac{1}{\tau_{2}} \frac{1}{\left(x_{0}-x\right)^{3}}\right) \frac{I}{I_{0}}+\frac{2 u}{\tau_{x} e^{u}},
$$

where $u=\left(x_{0}-x\right)^{2} / 2 \sigma_{x}^{2}, x_{0}$ is the position of the beam center in terms of the raw scraper coordinate, $\sigma_{x}$ the horizontal rms beam size, $\tau_{x}$ the horizontal damping time, $I$ and $I_{0}$ are the total beam current during the scan and the initial value, respectively. The fitting parameters are $\tau_{1,2}, x_{0}$ and $\sigma_{x}$ and the damping time is $\tau_{x}=3.38 \mathrm{~ms}$ for the low emittance lattice and $\tau_{x}=3.57 \mathrm{~ms}$ for the achromatic lattice when insertions devices are closed.

The experimental data and the fitted curve for the low emittance lattice are shown in Figure 9. Error bars for lifetime measurement are below $1 \%$. From fitting we obtained the rms beam size $\sigma_{x}=0.378 \pm 0.001 \mathrm{~mm}$, as compared to the calculated value $0.323 \mathrm{~mm}$ for the low emittance lattice. For the achromatic lattice, we obtain $\sigma_{x}=0.487 \pm 0.002 \mathrm{~mm}$ while the calculated value is $0.416 \mathrm{~mm}$. Although the measured beam size contains a systematic error of nearly $20 \%$ it still provides useful information for the scaling of the beam size under different lattice and ID configurations. 


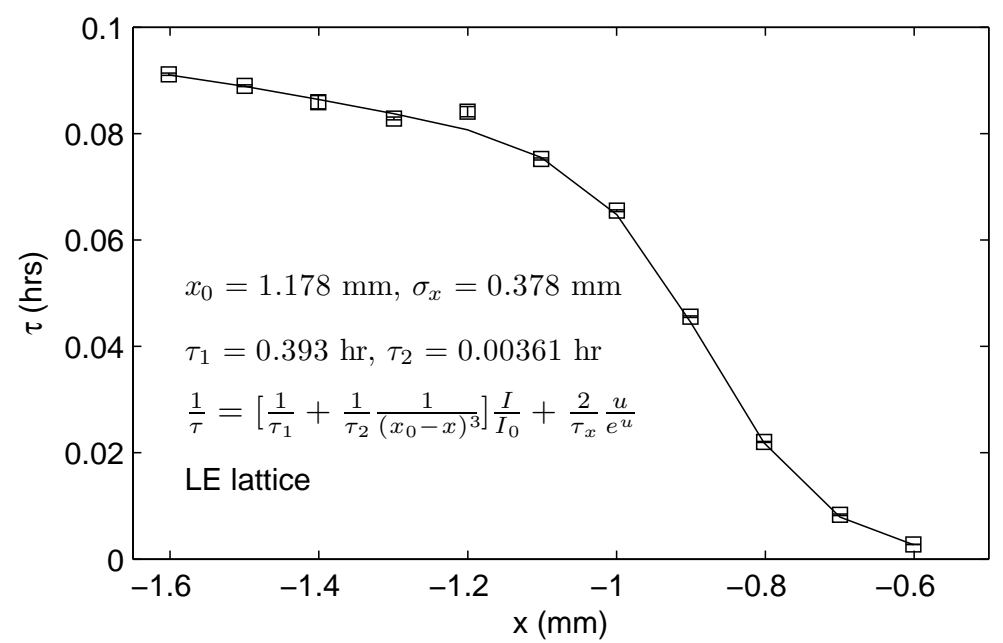

Figure 9: Lifetime vs horizontal scraper position for the low emittance lattice. There was $28 \mathrm{~mA}$ in a single bunch when the scan began.

\section{Conclusion}

Systematic lifetime studies have been conducted for the SPEAR3 storage ring. The gas scattering lifetime and Touschek lifetime were separated by filling the ring with various total currents and numbers of bunches. Coulomb lifetime was further separated using vertical scraper scans. The Touschek lifetime vs. rf gap voltage measurements were performed with the horizontal scraper inserted to several different positions. The measurements agree with calculations when the momentum aperture was obtained by tracking the calibrated lattice. Rf voltage scans were also made with the vertical scraper inserted. The data show that the motion of Touschek scattered particles is coupled to the vertical plane with an amplitude of about $0.7 \mathrm{~mm}$ with the horizontal scraper located at $\mathrm{x}=-15.5 \mathrm{~mm}$. This result is again consistent with tracking results from the coupled lattice model. The rf voltage scan with reduced vertical aperture may be a tool to further study nonlinear coupled motion.

Quantum lifetime was then measured with the rf gap voltage reduced. From these measurements we deduced the energy loss per revolution including the radiation energy loss from insertion devices. It is shown this technique can resolve changes in energy loss down to a few keV level. The measured radiation energy loss when all IDs are open agrees with calculations based 
on a dipole model that include the rectangular gradient dipole effect and the fringe field effect. Quantum lifetime was also measured with the horizontal scraper inserted to the vicinity of the beam core, from which the horizontal beam size was deduced. The data analysis tends to overestimate beam size by about $20 \%$ due to systematic errors of an unknown source. The quantum lifetime measurement of energy loss and beam size is nonetheless a useful technique for future experiments and machine characterization.

\section{Acknowledgment}

The authors thank J. Safranek for valuable discussions. The study is supported by DOE Contract No. DE-AC02-76SF00515.

\section{References}

[1] H. Wiedemann, Particle Accelerator Physics, 2nd edition, Springer, (1999).

[2] J. Corbett, et al, Short bunch measurement in SPEAR3, PAC09, Vancouver, Canada (2009)

[3] J. Safranek, Nucl. Instrum. Methods A 388 (1997), 1-2, 27.

[4] C. Steier, et al, Phys. Rev. E, 65, 056506 (2002).

[5] A. Nadji, et al, the PAC97 Proceedings, Vancouver, Canada (1997).

[6] M. Belgroune, et al, the PAC03 Proceedings, Portland, Oregon (2003).

[7] S. Y. Lee, Accelerator Physics, World Scientific (1999).

[8] X. Huang, J. Safranek, the IPAC10 Proceedings, Kyoto, Japan (2010). 